TRANSACTIONS OF THE

AMERICAN MATHEMATICAL SOCIETY

Volume 359, Number 10, October 2007, Pages 4695-4709

S 0002-9947(07)04132-3

Article electronically published on April 24, 2007

\title{
COMPATIBLE VALUATIONS AND GENERALIZED MILNOR $K$-THEORY
}

\author{
IDO EFRAT
}

\begin{abstract}
Given a field $F$ and a subgroup $S$ of $F^{\times}$there is a minimal group $S \leq H_{S} \leq F^{\times}$for which there exists an $S$-compatible valuation whose units are contained in $H_{S}$. Assuming that $S$ has finite index in $F^{\times}$and contains $\left(F^{\times}\right)^{p}$ for $p$ prime, we describe $H_{S}$ in computable $K$-theoretic terms.
\end{abstract}

\section{INTRODUCTION}

In many problems in field theory one needs to detect "arithmetically interesting" valuations on a field $F$, e.g., valuations $v$ satisfying some variant of Hensel's lemma. An especially useful such variant is $S$-compatibility: given a subgroup $S$ of the multiplicative group $F^{\times}$of $F$, one says that $v$ is $S$-compatible if the group $1+\mathfrak{m}_{v}$ of principal units of $v$ is contained in $S$ (here $\mathfrak{m}_{v}$ denotes the maximal ideal of the valuation ring $O_{v}$ of $v$ ). Since $S$-compatibility (as well as full Henselity) is always satisfied by the trivial valuation (for which $O_{v}=F$ ), some non-triviality condition is also needed. We therefore consider a second subgroup $H$ of $F^{\times}$and require that $O_{v}^{\times} \leq H$, where $O_{v}^{\times}$is the group of invertible elements in $O_{v}$. Let $\operatorname{Val}(S, H)$ be the set of all $S$-compatible valuations on $F$ which satisfy this requirement.

In practical problems, $S$ is usually given and one looks for an $S$-compatible valuation with $v\left(F^{\times}\right) / v(S)$ as large as possible. Equivalently, one looks for a subgroup $H$ which is as small as possible with $\operatorname{Val}(S, H) \neq \emptyset$. It follows from powerful results of Arason, Elman and Jacob [AEJ] (see also the related works [J], Wr1, [H] and E1]) that, apart from a well-understood exceptional case, there exists a canonical minimal group $H_{S}$ with these two properties. Thus $\operatorname{Val}\left(S, H_{S}\right) \neq \emptyset$, and $H_{S} \leq H$ whenever $\operatorname{Val}(S, H) \neq \emptyset$. Moreover, $\operatorname{Val}\left(S, H_{S}\right)$ then contains a canonical (coarsest) valuation.

A special case which has often been considered is when $S=\left(F^{\times}\right)^{p}$ for a prime number $p$ and $F$ contains a $p$ th root of unity. Then a valuation $v$ with residue characteristic $\neq p$ is $S$-compatible if and only if it is $p$-Henselian, i.e., Hensel's lemma holds for polynomials of degree $p$ Wd, Prop. 1.4]. Thus the problem in this case is to find a $p$-Henselian valuation $v$ on $F$ with $v\left(F^{\times}\right) / p v\left(F^{\times}\right) \cong F^{\times} / S O_{v}^{\times}$ as large as possible. This became especially important in the area of "birational anabelian geometry", where one recovers arithmetical properties of a field from the

Received by the editors March 24, 2005.

2000 Mathematics Subject Classification. Primary 19F99; Secondary 12J15, 19C99, 12J99.

This research was supported by the Israel Science Foundation grant No. 8008/02-1. 
knowledge of its absolute Galois group. The above-mentioned result of [AEJ] assures that the largest possible quotient $v\left(F^{\times}\right) / p v\left(F^{\times}\right)$which one can get is $F^{\times} / H_{S}$ (again, apart from the exceptional case). However, while $H_{S}$ can be described in elementary terms (see $\S 3$ ), when $p \neq 2$ it is a priori not clear how to detect it Galois-theoretically, as is required in such problems.

In this paper we solve this problem as follows. We consider a subgroup $\left(F^{\times}\right)^{p} \leq$ $S \leq F^{\times}$and associate to it a certain group $S \leq N_{S} \leq F^{\times}$which is defined in terms of the generalized Milnor $K$-ring functor $K_{*}^{M}(F) / S$, introduced in E5. We prove that when $\left(F^{\times}: S\right)<\infty$ (and apart from the exceptional case mentioned above) the groups $H_{S}$ and $N_{S}$ coincide. Now when $S=\left(F^{\times}\right)^{p}$ and $F$ contains a $p$ th root of unity, Kummer's theory and the Merkurjev-Suslin theorem imply that the ring $K_{*}^{M}(F) / S$ and the Galois cohomology ring $H^{*}\left(G_{F}(p), \mathbb{Z} / p\right)$ are isomorphic in degrees 1 and 2 (where $G_{F}(p)$ is the maximal pro- $p$ Galois group of $F$; see [MS], [JWd, $\S 1$, Ka $)$. Furthermore, $N_{S}$ is actually determined by the degree $\leq 2$ part of $K_{*}^{M}(F) / S$. Consequently, $G_{F}(p)$ indeed determines $N_{S}=H_{S}$ - in fact, in an effectively computable way.

This quantitative result strengthens the results of [HJ] and [Ko, which are of a qualitative nature - i.e., yield valuations $v$ with $v\left(F^{\times}\right) \neq p v\left(F^{\times}\right)$. It also improves the quantitative results of [E1, which gave another bound on the possible size of $v\left(F^{\times}\right) / p v\left(F^{\times}\right)$for $p$-Henselian valuations $v$. While the latter bound was sufficient for some applications ( $\mathrm{EF}$, [E2, E3]), it was not sharp, due to the non-canonical nature of the construction there.

As an application we go back in $\S 8$ to results of Bass-Tate BaT, Wadsworth Wd, and [E5], showing that the Milnor $K$-ring of an $S$-compatible valued field $(F, v)$ can be built from the corresponding $K$-ring of the residue field and the group $v\left(F^{\times}\right) / v(S)$ as an extension (this construction is analogous to the construction of polynomial rings in a set of variables over a given ring; see $\S 5)$. We prove a converse result, asserting that extension structures on $K_{*}^{M}(F) / S$ must originate from $S$-compatible valuations (Theorem 8.1).

The exceptional case mentioned above is that of totally rigid groups $S$. When $\left(F^{\times}\right)^{p} \leq S$ as before, this means in a $K$-theoretic language that $K_{*}^{M}(F) / S$ is an extension of either $K_{*}^{M}(\mathbb{R}) / 2$ (if $p=2$ ) or $K_{*}^{M}(\mathbb{C}) / p$. As we show in $\S 9$, totally rigid subgroups of the first kind are nothing but the fans, which were extensively studied in the context of ordered fields. Thus our combined $K$-theoretic/valuation-theoretic approach reveals a non-real analog of fans, namely, the totally rigid subgroups of the second kind.

\section{Valuations}

We first recall some basic facts about valuations which will be needed later on. All valuations will be in the sense of Krull, i.e., corresponding to valuation rings (see, e.g., [Bo, Ch. VI], $[\mathrm{En},[\mathrm{R}$ ), and we will not distinguish between valuations with equal valuation rings. Given valuations $v, u$ on a field $F$, we say that $v$ is finer than $u$ (and $u$ is coarser than $v$ ) if $O_{v} \subseteq O_{u}$. Other equivalent conditions are that $O_{v}^{\times} \leq O_{u}^{\times}, \mathfrak{m}_{u} \subseteq \mathfrak{m}_{v}$, or that there is an epimorphism of ordered abelian groups $\varphi: v\left(F^{\times}\right) \rightarrow u\left(F^{\times}\right)$such that $u=\varphi \circ v$. In this case we write $u \leq v$. This is a partial ordering on the set of all valuations on $F$. We say that $v$ and $u$ are comparable if one of them is coarser than the other. The collection of all 
valuations on $F$ has a tree structure with respect to $\leq$, with the trivial valuation as its minimum:

Proposition 2.1. The above partially ordered set has the following properties:

(a) if $v, u, u^{\prime}$ are valuations with $u \leq v$ and $u^{\prime} \leq v$ then $u, u^{\prime}$ are comparable;

(b) every collection $v_{i}, i \in I$, of valuations on $F$ has a finest common coarsening (i.e., an infimum).

Proof. For (a) see [Bo, Ch. VI, §4.1].

To prove (b) let $O$ be the subring of $F$ generated by the valuation ring $O_{v_{i}}, i \in I$. It is necessarily a valuation ring. The corresponding valuation is as required.

The next lemma is a special case of [E4, Cor. 2.3].

Lemma 2.2. Let $v_{1}, v_{2}$ be incomparable valuations on the field $F$ and let $u$ be their finest common coarsening. Then $O_{u}^{\times}=\left(1+\mathfrak{m}_{v_{1}}\right)\left(1+\mathfrak{m}_{v_{2}}\right)$.

Given subsets $C_{i}, i \in I$, of $F$ let $\prod_{i \in I} C_{i}$ be the set of all products $\prod_{i \in I} c_{i}$, where $c_{i} \in C_{i}$ for all $i$, and $c_{i}=1$ for all but finitely many $i$ (this makes sense also when $I$ is infinite).

Proposition 2.3. Let $v_{i}, i \in I$, be valuations on the field $F$ and let $u$ be their finest common coarsening. Then:

(a) $O_{u}=\prod_{i \in I} O_{v_{i}}$;

(b) $O_{u}^{\times}=\prod_{i \in I} O_{v_{i}}^{\times}$.

Proof. (a) We first show that $\prod_{i \in I} O_{v_{i}}$ is a ring. Indeed, consider elements $z=\prod_{i \in I} z_{i}$ and $t=\prod_{i \in I} t_{i}$ of $\prod_{i \in I} O_{v_{i}}$, with $z_{i}, t_{i} \in O_{v_{i}}$ as above. Clearly, $z t=\prod_{i \in I}\left(z_{i} t_{i}\right) \in \prod_{i \in I} O_{v_{i}}$. Also take an arbitrary $i_{0} \in I$. By the ultrametric inequality, $v_{i_{0}}(z+t) \geq \min \left\{v_{i_{0}}(z), v_{i_{0}}(t)\right\}-$ say, $v_{i_{0}}(z+t) \geq v_{i_{0}}(z)$. Then $z+t \in$ $z O_{i_{0}} \subseteq \prod_{i \in I} O_{v_{i}}$, as desired.

Since $\prod_{i \in I} O_{v_{i}}$ contains the valuation rings $O_{v_{i}}, i \in I$, it is itself a valuation ring. Obviously, it has no proper subrings containing all the $O_{v_{i}}, i \in I$.

(b) We first verify this in the case of two valuations $v_{1}, v_{2}$.

If $v_{1}, v_{2}$ are comparable, say, $v_{1} \leq v_{2}$, then $u=v_{1}$, so $O_{u}^{\times}=O_{v_{1}}^{\times} \geq O_{v_{2}}^{\times}$, and the assertion is clear.

If $v_{1}, v_{2}$ are incomparable, then Lemma 2.2 gives

$$
O_{u}^{\times}=\left(1+\mathfrak{m}_{v_{1}}\right)\left(1+\mathfrak{m}_{v_{2}}\right) \leq O_{v_{1}}^{\times} O_{v_{2}}^{\times} .
$$

On the other hand, $O_{v_{i}}^{\times} \leq O_{u}^{\times}, i=1,2$, so we are done once again.

The case of finitely many valuations $v_{i}$ follows from the case of two valuations by induction.

Finally consider the general case. Since $u$ is coarser than every $v_{i}$, we have $O_{u}^{\times} \geq \prod_{i \in I} O_{v_{i}}^{\times}$. Conversely, take $x \in O_{u}^{\times}$. (a) gives finite subsets $J, J^{\prime}$ of $I$ with $x \in \prod_{j \in J} O_{v_{j}}$ and $x^{-1} \in \prod_{j \in J^{\prime}} O_{v_{j}}$. We may replace both $J$ and $J^{\prime}$ by $J \cup J^{\prime}$ to assume that $J=J^{\prime}$. Let $w$ be the finest common coarsening of $v_{j}, j \in J$. By (a) again, $O_{w}=\prod_{j \in J} O_{v_{j}}$, and by what we have already seen, $O_{w}^{\times}=\prod_{j \in J} O_{v_{j}}^{\times}$. Hence $x \in O_{w}^{\times} \leq \prod_{i \in I} O_{v_{i}}^{\times}$, as desired.

Denote the residue field of a valuation $v$ on $F$ by $\bar{F}_{v}$. For a subgroup $S$ of $F^{\times}$ let $\bar{S}_{v}$ be the image of $S \cap O_{v}^{\times}$under the residue homomorphism $O_{v} \rightarrow \bar{F}_{v}$. It is a 
subgroup of $\bar{F}_{v}^{\times}$. When $v$ is $S$-compatible there is a canonical short exact sequence E5, (5.4)]

$$
1 \rightarrow \bar{F}_{v}^{\times} / \bar{S}_{v} \rightarrow F^{\times} / S \rightarrow v\left(F^{\times}\right) / v(S) \rightarrow 0 .
$$

If $u$ is another valuation on $F$ which is coarser than $v$, then $O_{v} / \mathfrak{m}_{u}$ is a valuation ring on $\bar{F}_{u}$. We denote the corresponding valuation by $v / u$. One has $\left(\bar{F}_{u}\right)_{v / u} \cong \bar{F}_{v}$ canonically, and this isomorphism identifies $\left(\bar{S}_{u}\right)_{v / u}$ with $\bar{S}_{v}$ (see Bo, Ch. VI, $\left.\S 4.3\right]$ ).

\section{Existence of VAluations}

In this section we summarize some of the main results of AEJ which will be required later on. We fix the field $F$ and consider subgroups $S \leq H$ of $F^{\times}$. We set:

$$
\begin{aligned}
O^{-}(S, H) & =(S-1) \backslash H, \\
O^{+}(S, H) & =\left\{x \in H \mid x O^{-}(S, H) \subseteq O^{-}(S, H)\right\}, \\
O(S, H) & =O^{-}(S, H) \cup O^{+}(S, H) .
\end{aligned}
$$

Remark 3.1. For $K$-theoretic purposes it is actually more convenient to define $O^{-}(S, H)$ as $(1-S) \backslash H$. Then the main results of [AEJ] remain valid with some minor sign adaptations (see E1]). However since we will extensively use here the results of $[\mathrm{AEJ}]$, we keep these definitions.

Let

$$
A_{S}=\left\{x \in F^{\times} \mid S-x S \nsubseteq S \cup-x S\right\}, \quad H_{S}=\left\langle-1, A_{S}\right\rangle .
$$

For $x \in S$ we have $0 \in S-x S$ but $0 \notin S \cup-x S$. Hence $S \subseteq A_{S}$. In the terminology of AEJ, $A_{S}$ consists of all $x \in F$ such that $-x$ is not $S$-rigid. An element $x$ of $F$ is called $S$-basic if $x \in A_{S} \cup-A_{S}$. Thus $H_{S}$ is the subgroup of $F^{\times}$generated by the $S$-basic elements. The pair $(S, H)$ is pre-additive if $1-O^{-}(S, H) O^{-}(S, H) \subseteq S$ (see [AEJ, Lemma 2.6]).

Theorem 3.2. The following conditions are equivalent:

(a) $\operatorname{Val}(S, H) \neq \emptyset$;

(b) $O(S, H)$ is a valuation ring corresponding to a valuation in $\operatorname{Val}(S, H)$ which is coarser than any other valuation in $\operatorname{Val}(S, H)$;

(c) $H_{S} \leq H$ and $(S, H)$ is pre-additive.

Proof. $(\mathrm{b}) \Rightarrow(\mathrm{a})$ : Trivial.

$(\mathrm{a}) \Rightarrow(\mathrm{c})$ : Let $v \in \operatorname{Val}(S, H)$. Obviously, $-1 \in O_{v}^{\times} \leq H$. By AEJ, Prop. 1.5(1)], $A_{S} \subseteq S O_{v}^{\times} \leq H$.

Finally, if $x \in O^{-}(S, H)$, then $1+x \in S \leq H$, as well as $x \notin H$. Therefore $1+x^{-1}=x^{-1}(1+x) \notin H$, and in particular, $1+x^{-1} \notin S$. Since $v$ is $S$-compatible, $x^{-1} \notin \mathfrak{m}_{v}$, so $x \in O_{v} \backslash H$. Conclude that

$$
O^{-}(S, H) \subseteq O_{v} \backslash H \subseteq O_{v} \backslash O_{v}^{\times}=\mathfrak{m}_{v},
$$

whence

$$
1-O^{-}(S, H) O^{-}(S, H) \subseteq 1-\mathfrak{m}_{v} \mathfrak{m}_{v} \subseteq 1+\mathfrak{m}_{v} \leq S .
$$

$(\mathrm{c}) \Rightarrow(\mathrm{b})$ : By (c), $H$ contains the $S$-basic elements of $F$. Therefore AEJ, Th. 2.10] shows that $O(S, H)$ corresponds to a valuation in $\operatorname{Val}(S, H)$. By [AEJ, Prop. $3.2]$, it is coarser than any other valuation in $\operatorname{Val}(S, H)$. 
The picture given by Theorem 3.2 would be complete if we knew that $\operatorname{Val}\left(S, H_{S}\right)$ is non-empty. While this is not always the case, the following proposition shows that the exceptions are quite rare.

Proposition 3.3. If $\operatorname{Val}\left(S, H_{S}\right)=\emptyset$, then

(a) $H_{S}=\langle-1, S\rangle$;

(b) there exists an intermediate group $H_{S}<L \leq F^{\times}$such that $\left(L: H_{S}\right)=2$ and $\operatorname{Val}(S, L) \neq \emptyset$;

(c) $-1 \in S$ or $S+S \subseteq S$;

(d) $A_{S}=S$.

Proof. By assumption, $O\left(S, H_{S}\right)$ does not correspond to a valuation in $\operatorname{Val}\left(S, H_{S}\right)$. Therefore (a)-(c) follow from [AEJ, Th. 2.16].

For (d), suppose that $x \in A_{S} \backslash S$. Then (a) implies that $-x \in S$. Hence $-1 \notin S$, so by (c), $S-x S=S+S \subseteq S=S \cup-x S$. This contradicts $x \in A_{S}$.

\section{4. $\kappa$-STRUCTURES}

We recall from [E5] the basic notions of the category of $\kappa$-structures, which serves as the target category for the generalized Milnor $K$-ring functor.

Denote the tensor algebra of an abelian group $\Gamma$ (considered as a $\mathbb{Z}$-algebra) by Tens $(\Gamma)$. Let $\kappa=\bigoplus_{r=0}^{\infty} \kappa_{r}=\operatorname{Tens}(\{ \pm 1\})$, and let $\epsilon$ be the non-trivial element of $\kappa_{1} \cong\{ \pm 1\}$. A $\kappa$-structure consists of a graded ring $A=\bigoplus_{r=0}^{\infty} A_{r}$ and a graded ring homomorphism $\kappa \rightarrow A$ such that:

(i) $A_{0}=\mathbb{Z}$ and the homomorphism $\kappa \rightarrow A$ is the identity in degree 0 ;

(ii) $A_{1}$ generates $A$ as a ring;

(iii) the image $\epsilon_{A}$ of $\epsilon$ in $A_{1}$ satisfies $a^{2}=\epsilon_{A} a=a \epsilon_{A}$ for all $a \in A_{1}$.

For $a, b \in A_{1}$, (iii) gives $a b+b a=(a+b)^{2}-a^{2}-b^{2}=0$, so $A$ is anti-commutative. The trivial $\kappa$-structure $\mathbf{0}$ is defined by $\mathbf{0}_{0}=\mathbb{Z}$ and $\mathbf{0}_{r}=0$ for $r \geq 1$. A morphism $A \rightarrow B$ of $\kappa$-structures is a graded ring homomorphism which commutes with the structural homomorphisms $\kappa \rightarrow A, \kappa \rightarrow B$.

The tensor product in the category of graded rings is defined as usual by

$$
A \otimes_{\mathbb{Z}} B=\bigoplus_{r=0}^{\infty}\left(\bigoplus_{i+j=r} A_{i} \otimes_{\mathbb{Z}} B_{j}\right),
$$

with the multiplication law

$$
(a \otimes b)\left(a^{\prime} \otimes b^{\prime}\right)=(-1)^{i^{\prime} j} a a^{\prime} \otimes b b^{\prime}
$$

for $a \in A_{i}, a^{\prime} \in A_{i^{\prime}}, b \in B_{j}, b^{\prime} \in B_{j^{\prime}}$. Given $\kappa$-structures $A, B$, we define their tensor product in the category of $\kappa$-structures to be $A \otimes_{\kappa} B=\left(A \otimes_{\mathbb{Z}} B\right) / I$, where $I$ is the homogeneous ideal generated by $\epsilon_{A} \otimes 1_{B}-1_{A} \otimes \epsilon_{B}$. The structural homomorphism $\kappa \rightarrow A \otimes_{\kappa} B$ is given by $\epsilon \mapsto \epsilon_{A} \otimes 1_{B}+I=1_{A} \otimes \epsilon_{B}+I$.

Next, for an abelian group $\Gamma$ let $\kappa[\Gamma]$ be the quotient of $\operatorname{Tens}\left(\kappa_{1} \oplus \Gamma\right)$ by the homogeneous ideal generated by all elements $\epsilon \otimes \gamma-\gamma \otimes \gamma$, where $\gamma \in \Gamma$. The obvious embedding $\kappa_{1} \hookrightarrow \kappa_{1} \oplus \Gamma$ induces a graded ring homomorphism $\kappa \rightarrow \kappa[\Gamma]$, making $\kappa[\Gamma]$ into a $\kappa$-structure. More generally, for a $\kappa$-structure $\bar{A}$ we define the extension $\bar{A}[\Gamma]=\bar{A} \otimes_{\kappa} \kappa[\Gamma]$ of $\bar{A}$ by $\Gamma$. One may identify $(\bar{A}[\Gamma])_{1}=\bar{A}_{1} \oplus \Gamma$. If $\Gamma^{\prime}$ is another abelian group, then $(\bar{A}[\Gamma])\left[\Gamma^{\prime}\right] \cong \bar{A}\left[\Gamma \oplus \Gamma^{\prime}\right]$ canonically [E5, Cor. 1.3]. 
4.1 Examples. (a) $\mathbf{0}[\Gamma]$ is just the alternating $\mathbb{Z}$-algebra over $\Gamma \underline{\mathrm{Lg}}$, Ch. XVI, $\S 6]$.

(b) Let $\bar{A}$ be a $\kappa$-structure, let $\Gamma=\langle\gamma\rangle$ be a cyclic group, and let $A=\bar{A}[\Gamma]$. For $r \geq 1$, the group $A_{r}$ is generated by the image of $\bigoplus_{i+j=r}\left(\bar{A}_{i} \otimes_{\mathbb{Z}} \Gamma^{\otimes j}\right)$. By (iii) above, $\gamma^{j}=\epsilon_{\bar{A}}^{j-1} \gamma$ in $A$. It follows that $A_{r}=\bar{A}_{r} \oplus\left(\bar{A}_{r-1} \otimes_{\mathbb{Z}} \Gamma\right)$. Under this identification, the product $A_{1} \times A_{1} \rightarrow A_{2}$ is given by

$$
(\bar{a}, i \gamma) \cdot(\bar{b}, j \gamma)=\left(\bar{a} \cdot \bar{b},\left(j \bar{a}-i \bar{b}+i j \epsilon_{\bar{A}}\right) \otimes \gamma\right)
$$

for $\bar{a}, \bar{b} \in \bar{A}_{1}$ and $i, j \in \mathbb{Z}$.

The construction of extensions is intimately related to the following quite subtle notion, which goes back to Szymiczek [S] and Ware (Wr1], Wr2]).

Definition. Let $n$ be a positive integer, and let $A$ be a $\kappa$-structure. We say that $a \in A_{1}$ is $n$-rigid if:

(1) $\langle a\rangle \cong \mathbb{Z} / n$; and

(2) for every $b \in A_{1}$ with $a \cdot b=0$ in $A_{2}$ there exist integers $i, j$, at least one of which is prime to $n$, such that $j\left(\epsilon_{A}+a\right)=i b$.

Note that when $n=p$ is prime and $p A_{1}=0,(2)$ means that for every $b \in A_{1}$ with $a \cdot b=0$ the elements $\epsilon_{A}+a$ and $b$ of $A_{1}$ are $\mathbb{F}_{p}$-linearly dependent. Also note that when $p \neq 2$ we actually have $\epsilon_{A}=0$. The first fundamental connection between rigidity and extensions is the following fact.

Proposition 4.2. Let $n$ be a positive integer, and let $\bar{A}$ be a $\kappa$-structure such that $n \bar{A}_{1}=0$. Let $\Gamma$ be a free $\mathbb{Z} / n$-module and let $A=\bar{A}[\Gamma]$. Suppose that the projection of $a \in A_{1}$ to $\Gamma$ has order $n$. Then a is n-rigid.

Proof. We may decompose $\Gamma=\Gamma_{1} \oplus \Gamma_{2}$, where $\Gamma_{2} \cong \mathbb{Z} / n$ and where the projection of $a$ in $\Gamma_{2}$ has order $n$. As $A \cong\left(\bar{A}\left[\Gamma_{1}\right]\right)\left[\Gamma_{2}\right]$, it is enough to consider the case where $\Gamma=\Gamma_{2}=\langle\gamma\rangle$ is cyclic of order $n$. Then the computations of Example 4.1(b) apply.

We write $a=(\bar{a}, i \gamma) \in A_{1}=\bar{A}_{1} \oplus \Gamma$, with $\bar{a} \in \bar{A}_{1}$ and $i \in \mathbb{Z}$. By assumption, $\langle a\rangle \cong \mathbb{Z} / n$, and furthermore, $n$ and $i$ are relatively prime. Note that if $\epsilon_{\bar{A}} \neq 0$, then $n$ is even, so $i$ is odd and $i \epsilon_{\bar{A}}=\epsilon_{\bar{A}}$. Obviously, this also holds when $\epsilon_{\bar{A}}=0$.

Now let $b=(\bar{b}, j \gamma) \in A_{1}=\bar{A}_{1} \oplus \Gamma$ and suppose that $a \cdot b=0$ in $A_{2}$. By (4.1), $i \bar{b}=j \bar{a}+i j \epsilon_{\bar{A}}=j\left(\bar{a}+\epsilon_{\bar{A}}\right)$. Therefore

$$
i b=(i \bar{b}, i j \gamma)=j\left(\bar{a}+\epsilon_{\bar{A}}, i \gamma\right)=j\left(a+\epsilon_{A}\right),
$$

as required.

4.3 Examples. (1) Let $p$ be a prime number and let $\Gamma$ be an abelian group of exponent $p$. Let $A=\mathbf{0}[\Gamma]$. By Proposition 4.2, every non-zero element of $A_{1}$ is $p$-rigid.

(2) Let $\Gamma$ be an abelian group of exponent 2 and let $A=\kappa[\Gamma]$. Then every nonzero element $a$ of $A_{1}$ is 2-rigid. Indeed, for $a=\epsilon_{A}$ this is trivial from the definition of rigidity, while for $a \neq 0, \epsilon_{A}$ it follows again from Proposition 4.2 .

Given a $\kappa$-structure $A$ we define a homomorphism $\operatorname{Bock}_{A}: A_{1} \rightarrow A_{2}$ by

$$
\operatorname{Bock}_{A}(a)=a^{2}=\epsilon_{A} \cdot a .
$$

Generalizing standard terminology (see, e.g., NSW, p. 191]), we call Bock $_{A}$ the Bockstein map of $A$. 
Lemma 4.4. Let $\Gamma$ be an abelian group of exponent 2 , and let $A=\kappa[\Gamma]$. Then Bock $_{A}$ is injective.

Proof. Suppose that $0 \neq a \in A_{1}$ satisfies $\operatorname{Bock}_{A}(a)=0$. As we have just seen, $a$ is 2-rigid. Since $a^{2}=0$, the elements $a$ and $a+\epsilon$ of $A_{1}$ must be $\mathbb{F}_{2}$-linearly

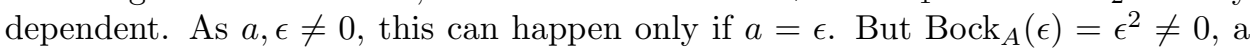
contradiction.

\section{Generalized Milnor $K$-Rings}

Let $F$ be a field and $S$ a subgroup of $F^{\times}$. For $r \geq 0$ let $\operatorname{St}_{F, r}(S)$ be the subgroup of $\left(F^{\times} / S\right)^{\otimes r}=F^{\times} \otimes_{\mathbb{Z}} \cdots \otimes_{\mathbb{Z}} F^{\times}(r$ times $)$ generated by all elements $a_{1} S \otimes \cdots \otimes a_{r} S$ such that $1 \in a_{i} S+a_{j} S$ for some $i \neq j$. Generalizing standard terminology, we call such generators Steinberg elements. As in [E5] we set

$$
K_{r}^{M}(F) / S=\left(F^{\times} / S\right)^{\otimes r} / \operatorname{St}_{F, r}(S) .
$$

Then $K_{*}^{M}(F) / S=\bigoplus_{r=0}^{\infty} K_{r}^{M}(F) / S$ is a $\kappa$-structure with respect to the multiplication induced from the tensor algebra over $F^{\times}$, and with the structural morphism $\kappa \rightarrow K_{*}^{M}(F) / S$ given by $\epsilon \mapsto-S \in F^{\times} / S$ (see [E5, §2]). We call it the Milnor $K$-ring of $F$ modulo $S$. In particular, $K_{0}^{M}(F) / S=\mathbb{Z}$ and $K_{1}^{M}(F) / S=F^{\times} / S$. When $S=\{1\}$ this is the classical Milnor $K$-ring $K_{*}^{M}(F)$ defined in Mi. When $S=\left(F^{\times}\right)^{m}$ for a positive integer $m$, the rings $K_{*}^{M}(F) / S$ and $K_{*}^{M}(F) / m$ coincide except in degree 0 .

Given $a_{1}, \ldots, a_{r} \in F^{\times}$we denote the image of $a_{1} S \otimes \cdots \otimes a_{r} S$ in $K_{r}^{M}(F) / S$ by $\left\{a_{1}, \ldots, a_{r}\right\}_{S}$. The map $\left\{a_{1}, \ldots, a_{r}\right\}_{S}$ is multi-linear and anti-commutative. Also, since the identity $\{a,-a\}=0$ for $a \in F^{\times}$is well-known to hold in $K_{2}^{M}(F)$ [Mi, §1], one has $\{a,-a\}_{S}=0$ in $K_{*}^{M}(F) / S$. Therefore $\{a, a\}_{S}=\{-1, a\}_{S}=\{a,-1\}_{S}$.

For an intermediate group $S \leq S^{\prime} \leq F^{\times}$there is a canonical restriction morphism

$$
\text { Res: } K_{*}^{M}(F) / S \rightarrow K_{*}^{M}(F) / S^{\prime},
$$

which maps a generator $\left\{a_{1}, \ldots, a_{r}\right\}_{S}$ of $K_{r}^{M}(F) / S$ to $\left\{a_{1}, \ldots, a_{r}\right\}_{S^{\prime}}$.

The $S$-compatible valuations reflect in the $K$-ring as extensions, in the sense of $\S 4$. This is the following special case of [E5, Th. 5.1], which in turn extends earlier results of Bass and Tate [BaT, I, Prop. 4.3] and Wadsworth [Wd, §2].

Theorem 5.1. Let $F$ be a field, $p$ a prime number, and $\left(F^{\times}\right)^{p} \leq S \leq F^{\times}$an intermediate group. Let $v$ be an $S$-compatible valuation. Then

$$
K_{*}^{M}(F) / S \cong\left(K_{*}^{M}\left(\bar{F}_{v}\right) / \bar{S}_{v}\right)\left[v\left(F^{\times}\right) / v(S)\right] .
$$

The arithmetic structure of $F$ as an ordered field is also reflected in its $K$ theory in a rather simple way as follows. A subgroup $S$ of $F^{\times}$is called a (proper) preordering if it is additively closed, contains $\left(F^{\times}\right)^{2}$, but does not contain -1 . It is called an ordering if in addition $F^{\times}=S \cup-S$.

Proposition 5.2. $\quad$ (a) $S$ is an ordering if and only if $K_{*}^{M}(F) / S \cong \kappa$ as $\kappa$ structures.

(b) For distinct orderings $S_{1}, S_{2}$ on $F$ one has $K_{*}^{M}(F) /\left(S_{1} \cap S_{2}\right) \cong \kappa[\mathbb{Z} / 2]$.

Proof. (a) See [E5, Prop. 3.2].

(b) Set $S=S_{1} \cap S_{2}$ and choose $a_{1} \in S_{1} \backslash S_{2}$. Then $a_{2}:=-a_{1} \in S_{2} \backslash S_{1}$, and $F^{\times} / S$ consists of the cosets of $1,-1, a_{1}, a_{2}$. Since $K_{*}^{M}(F) / S$ is a $\kappa$-structure, 
$\left\{a_{i}, a_{i}\right\}_{S}=\left\{-1, a_{i}\right\}_{S}, i=1,2$, and $\left\{a_{1}, a_{2}\right\}_{S}=0$. It follows that for $r \geq 2$ the group $K_{r}^{M}(F) / S$ consists only of the elements

$$
0,\{-1,-1, \ldots,-1\}_{S} \quad, \quad\left\{-1, \ldots,-1, a_{1}\right\}_{S} \quad, \quad\left\{-1, \ldots,-1, a_{2}\right\}_{S} .
$$

Furthermore, consider the restriction morphism Res: $K_{*}^{M}(F) / S \rightarrow K_{*}^{M}(F) / S_{i}(\cong$ $\kappa$, by (a)). When $i=1$ it maps only the first and the third elements in this list trivially, and when $i=2$ it maps only the first and the fourth elements trivially. It follows that these four elements are distinct.

On the other hand, Examples 4.1(b) shows that $(\kappa[\mathbb{Z} / 2])_{r}$ is a group of order 4 generated by $\epsilon^{r}$ and $\epsilon^{r-1} \gamma$, where $\gamma$ is the generator of $\mathbb{Z} / 2$. The multiplicative structure of $\kappa[\mathbb{Z} / 2]$ is then determined by the equality $\gamma^{2}=\epsilon \gamma$. Consequently the $\kappa$-structures $K_{*}^{M}(F) / S$ and $\kappa[\mathbb{Z} / 2]$ are isomorphic (with $a_{1}$ corresponding to $\gamma$ ).

\section{TOTALly RIGID SUBgROUPS}

Let $F$ be a field and $S$ a subgroup of $F^{\times}$. The identity $\{a,-a\}_{S}=0$ implies that every tensor product $a_{1} S \otimes \cdots \otimes a_{r} S$ such that $a_{1}, \ldots, a_{r} \in F^{\times}$and $a_{i} S=-a_{j} S$ for some $1 \leq i<j \leq r$ is contained in $\operatorname{St}_{F, r}(S)$. We say that $S$ is totally rigid if for every $r$ the group $\operatorname{St}_{F, r}(S)$ is in fact generated by such tensor products.

Proposition 6.1. $\quad$ (a) Assume that $-1 \in S$. Then $S$ is totally rigid if and only if $K_{*}^{M}(F) / S \cong \mathbf{0}[\Gamma]$ for some abelian group $\Gamma$.

(b) Assume that $-1 \notin S$ and $\left(F^{\times}\right)^{2} \leq S$. Then $S$ is totally rigid if and only if $K_{*}^{M}(F) / S \cong \kappa[\Gamma]$ for some group $\Gamma$.

Proof. (a) This follows from Examples 4.1(a), taking $\Gamma=F^{\times} / S$.

(b) This follows from the definition of $\kappa[\Gamma]$, where $\Gamma$ is taken to be a complement of the subgroup generated by $-S$ in $F^{\times} / S$.

Proposition 6.2. If $A_{S}=S$, then $S$ is totally rigid.

Proof. Let $a_{1} S \otimes \cdots \otimes a_{r} S$ be a standard generator of $\operatorname{St}_{F, r}(S)$, i.e., $1=a_{i} s+a_{j} s^{\prime}$ for some $i \neq j$ and some $s, s^{\prime} \in S$. If $a_{i} \in S$ or $a_{j} \in S$, then this generator is trivial. If $a_{i}, a_{j} \notin S=A_{S}$, then $a_{j} s^{\prime}=1-a_{i} s \in S-a_{i} S \subseteq S \cup-a_{i} S$ and $a_{j} s^{\prime} \notin S$. Hence $a_{j} S=-a_{i} S$.

We can now give a $K$-theoretic answer to the problem considered in $\S 3$ whether $\operatorname{Val}\left(S, H_{S}\right)$ is non-empty.

Corollary 6.3. Suppose that $S$ is not totally rigid. Then $\operatorname{Val}\left(S, H_{S}\right) \neq \emptyset$.

Proof. Apply Proposition 6.2 and Proposition 3.3(d).

From now on we fix a prime number $p$ and assume that $\left(F^{\times}\right)^{p} \leq S \leq F^{\times}$. Let

$$
T_{S}=\left\{\begin{array}{l|l}
x \in F^{\times} & 1-x \notin \bigcup_{i=0}^{p-1} x^{i} S
\end{array}\right\} .
$$

6.4 Remarks. (1) When $-1 \in S$ one has $T_{S} \subseteq A_{S}$.

(2) Suppose that $T_{S} \subseteq S$. Then $\operatorname{St}_{F, r}(S)$ is generated by the tensor products $a_{1} S \otimes \cdots \otimes a_{r} S$ such that $a_{i} S=a_{j} S$ for some $1 \leq i<j \leq r$. If in addition $-1 \in S$, then we deduce that $S$ is totally rigid. 
We now define the group $N_{S}$ as follows: it is the subgroup of $F^{\times}$generated by $-1, S$, and by all $x \in F^{\times}$such that $\{x\}_{S}$ is not $p$-rigid in $K_{*}^{M}(F) / S$. More specifically, $N_{S}$ is generated by $-1, S$, and by all $x \in F^{\times}$for which there exists $y \in F^{\times}$such that $\{x, y\}_{S}=0$ and $y \notin \bigcup_{i=0}^{p-1}(-x)^{i} S$.

Lemma 6.5. (a) If $-1 \in S$, then $T_{S} \subseteq N_{S}$.

(b) If $p=2$, then $A_{S} \subseteq N_{S}$.

Proof. (a) Take $1 \neq x \in T_{S}$. Then $\{x, 1-x\}=0$ and

$$
1-x \notin \bigcup_{i=0}^{p-1} x^{i} S=\bigcup_{i=0}^{p-1}(-x)^{i} S,
$$

so $x \in N_{S}$.

(b) Let $x \in A_{S}$. Thus $S-x S \nsubseteq S \cup-x S$. Hence there exists $x^{\prime} \in x S$ such that $y=1-x^{\prime} \notin S \cup-x^{\prime} S$. Conclude that $x^{\prime} \in N_{S}$, whence also $x \in N_{S}$.

We will also need the following estimate:

Proposition 6.6. If $-1 \in S$ and $T_{S} \subseteq S$, then $\left(H_{S}: S\right) \mid p$.

Proof. When the pair $\left(S, H_{S}\right)$ is pre-additive this is proved in [E1, Cor. 3.3] (following [HJ]).

When $\left(S, H_{S}\right)$ is not pre-additive, $\operatorname{Val}\left(S, H_{S}\right)=\emptyset$ by Theorem 3.2. Conclude from Proposition 3.3 that $A_{S}=S$, so $H_{S}=S$ and we are done once again.

Proposition 6.7. For $S$ totally rigid:

(a) $N_{S}=\langle-1, S\rangle$;

(b) $\left(H_{S}: S\right) \mid p$.

Proof. (a) By Examples 4.3(1), (2) and Proposition 6.1 all non-zero elements of $F^{\times} / S$ are $p$-rigid in $K_{*}^{M}(F) / S$. The assertion follows.

(b) Assume first that $-1 \in S$. Then $N_{S}=S$ by (a), so by Lemma 6.5(a), $T_{S} \subseteq S$. Now use Proposition 6.6.

Next assume that $-1 \notin S$. As $\left(F^{\times}\right)^{p} \leq S$ necessarily $p=2$. By Lemma $6.5(\mathrm{~b}), A_{S} \subseteq N_{S}$. Use (a) once again to conclude that $H_{S}=\langle-1, S\rangle$, whence $\left(H_{S}: S\right)=2$.

\section{Rigidity AND VALUATiOns}

Let again $p$ be a prime number and $\left(F^{\times}\right)^{p} \leq S \leq F^{\times}$an intermediate group. The subgroup $N_{S}$ gives a bound on the possible sizes of the quotients $v\left(F^{\times}\right) / v(S)$, with $v$ an $S$-compatible valuation:

Proposition 7.1. For every $S$-compatible valuation $v$ on $F$ one has $N_{S} \leq S O_{v}^{\times}$ and $\left(v\left(F^{\times}\right): v(S)\right) \leq\left(F^{\times}: N_{S}\right)$.

Proof. By Theorem 5.1, $K_{*}^{M}(F) / S \cong\left(K_{*}^{M}\left(\bar{F}_{v}\right) / \bar{S}_{v}\right)\left[v\left(F^{\times}\right) / v(S)\right]$ as $\kappa$-structures. By Proposition 4.2, elements of $F^{\times} / S$ whose projection via $v$ in $v\left(F^{\times}\right) / v(S)$ is nonzero are $p$-rigid in $K_{*}^{M}(F) / S$. Conclude from the definition of $N_{S}$ that $v\left(N_{S}\right) \leq$ $v(S)$, i.e., $N_{S} \leq S O_{v}^{\times}$. As $S \leq N_{S}$ we actually have $v(S)=v\left(N_{S}\right)$. Consequently,

$$
\left(v\left(F^{\times}\right): v(S)\right)=\left(v\left(F^{\times}\right): v\left(N_{S}\right)\right)=\left(F^{\times}: N_{S} O_{v}^{\times}\right) \leq\left(F^{\times}: N_{S}\right) .
$$


In Theorem 3.2 we have seen that if $S \leq H \leq F^{\times}$and $\operatorname{Val}(S, H) \neq \emptyset$, then $H_{S} \leq H$. Proposition 7.1 implies that the subgroup $N_{S}$ has the same property:

Corollary 7.2. If $S \leq H \leq F^{\times}$and $\operatorname{Val}(S, H) \neq \emptyset$, then $N_{S} \leq H$.

Again, this raises the question whether $\operatorname{Val}\left(S, N_{S}\right) \neq \emptyset$. This will be shown (under some assumptions) as a part of Theorem 7.5 below. We will first need the following key connection between the structure of $K_{*}^{M}(F) / S$ and valuations.

Lemma 7.3. Suppose that $\langle-1, S\rangle \leq M<N_{S}$ and $\left(N_{S}: M\right)=p$. Then:

(a) $T_{M} \subseteq M$;

(b) there exists $M \leq L \leq F^{\times}$such that $(L: M) \mid p$ and $\operatorname{Val}(M, L) \neq \emptyset$.

Proof. (a) Take $x \notin M$. We need to show that the cosets $\{x\}_{M}$ and $\{1-x\}_{M}$ are $\mathbb{F}_{p}$-linearly dependent in $F^{\times} / M$.

If $\{x\}_{S}$ and $\{1-x\}_{S}$ are both not $p$-rigid in $K_{*}^{M}(F) / S$, then $x, 1-x \in N_{S}$. Since $\operatorname{dim}_{\mathbb{F}_{p}}\left(N_{S} / M\right) \leq 1$, the cosets $\{x\}_{M}$ and $\{1-x\}_{M}$ are then $\mathbb{F}_{p}$-linearly dependent in $N_{S} / M\left(\leq F^{\times} / M\right)$, and we are done.

Next suppose that at least one of $\{x\}_{S},\{1-x\}_{S}$ is $p$-rigid in $K_{*}^{M}(F) / S$. From $\{x, 1-x\}_{S}=0$ we then deduce that either $\{-x\}_{S},\{1-x\}_{S}$ or $\{x\}_{S},\{-(1-x)\}_{S}$ are $\mathbb{F}_{p}$-linearly dependent in $F^{\times} / S$. But $-1 \in M$, so in both cases, $\{x\}_{M}$ and $\{1-x\}_{M}$ are $\mathbb{F}_{p^{-}}$-linearly dependent in $F^{\times} / M$.

(b) By (a) and Proposition 6.6, $\left(H_{M}: M\right) \mid p$. When $\operatorname{Val}\left(M, H_{M}\right) \neq \emptyset$ we can therefore take $L=H_{M}$.

When $\operatorname{Val}\left(M, H_{M}\right)=\emptyset$ Proposition 3.3 shows that $H_{M}=M, p=2$, and there is a subgroup $L$ as required.

The next main step towards the proof of Theorem 7.5 is the following proposition.

Proposition 7.4. Suppose that $-1 \in S<N_{S}$ and $\left(F^{\times}: S\right)<\infty$. Then there exists an $S$-compatible valuation $v$ on $F$ such that $N_{S}=S O_{v}^{\times}$.

Proof. Let $\mathcal{M}$ be the collection of all intermediate groups $S \leq M<N_{S}$ with $\left(N_{S}: M\right)=p$. The assumptions imply that $\mathcal{M}$ is finite and non-empty. For every $M \in \mathcal{M}$ Lemma $7.3(\mathrm{~b})$ yields a group $M \leq L_{M} \leq F^{\times}$with $\left(L_{M}: M\right) \mid p$ and a valuation $v_{M} \in \operatorname{Val}\left(M, L_{M}\right)$. Let $v$ be the finest common coarsening of the valuations $v_{M}, M \in \mathcal{M}$ (Proposition 2.1(b)). Then

$$
1+\mathfrak{m}_{v} \leq \bigcap_{M \in \mathcal{M}}\left(1+\mathfrak{m}_{v_{M}}\right) \leq \bigcap_{M \in \mathcal{M}} M=S
$$

so $v$ is $S$-compatible. By Proposition $7.1, N_{S} \leq S O_{v}^{\times}$. To show that this is an equality we distinguish between two cases.

Case I: For each $M \in \mathcal{M}$ there exists $M^{\prime} \in \mathcal{M}$ such that $v_{M}$ and $v_{M^{\prime}}$ are incomparable. For each $M$ we choose such $M^{\prime}$ and denote the finest common coarsening of $v_{M}$ and $v_{M}$ by $u_{M}$. Then $v$ is also the finest common coarsening of the $u_{M}$ for $M \in \mathcal{M}$. As $M \neq M^{\prime}$ Lemma 2.2 gives:

$$
O_{u_{M}}^{\times}=\left(1+\mathfrak{m}_{v_{M}}\right)\left(1+\mathfrak{m}_{v_{M^{\prime}}}\right) \leq M M^{\prime}=N_{S} .
$$

Conclude from Proposition 2.3(b) that $O_{v}^{\times}=\prod_{M \in \mathcal{M}} O_{u_{M}}^{\times} \leq N_{S}$, whence $N_{S}=$ $S O_{v}^{\times}$in this case. 
Case II: There exists $M \in \mathcal{M}$ such that $v_{M}$ is comparable to $v_{M^{\prime}}$ for all $M^{\prime} \in \mathcal{M}$. Then $v$ is the finest common coarsening of all the $v_{M^{\prime}}$ which are coarser than $v_{M}$. These $v_{M^{\prime}}$ form a chain with respect to coarsening (Proposition 2.1(a)). Since $\mathcal{M}$ is finite, $v=v_{M^{\prime}}$ for some $M^{\prime}$. Hence $O_{v}^{\times}=O_{v_{M^{\prime}}}^{\times} \leq L_{M^{\prime}}$. Therefore $M^{\prime}<N_{S} \leq$ $S O_{v}^{\times} \leq L_{M^{\prime}}$. Since $\left(L_{M^{\prime}}: M^{\prime}\right) \mid p$ this implies that $N_{S}=S O_{v}^{\times}$in this case as well.

We now come to our main result:

Theorem 7.5. Suppose that $\left(F^{\times}\right)^{p} \leq S \leq F^{\times}$and that $S$ is not totally rigid. Moreover, assume that $p=2$ or $\left(F^{\times}: S\right)<\infty$. Then:

(a) $\operatorname{Val}\left(S, N_{S}\right) \neq \emptyset$;

(b) $N_{S}=H_{S}$.

Proof. (a) We break the proof into three cases.

Case I: $p=2$. Then $A_{S} \subseteq N_{S}$, by Lemma 6.5(b). Hence $H_{S} \leq N_{S}$. Since $S$ is not totally rigid, Corollary 6.3 gives $\emptyset \neq \operatorname{Val}\left(S, H_{S}\right) \subseteq \operatorname{Val}\left(S, N_{S}\right)$.

Case II: $p \neq 2$ and $S<N_{S}$. Then $-1 \in\left(F^{\times}\right)^{p} \leq S$ and $\left(F^{\times}: S\right)<\infty$. We may therefore apply Proposition 7.4.

Case III: $p \neq 2$ and $S=N_{S}$. Then again $-1 \in S$, so by Lemma 6.5(a), $T_{S} \subseteq N_{S}=$ $S$. But according to Remarks 6.4(2) this can happen only when $S$ totally rigid, a contradiction.

(b) Corollary 6.3 gives again $\operatorname{Val}\left(S, H_{S}\right) \neq \emptyset$, so by Corollary $7.2, N_{S} \leq H_{S}$. On the other hand, (a) and Theorem 3.2 show that $H_{S} \leq N_{S}$.

Corollary 7.6. Under the assumptions of Theorem 7.5, there is an S-compatible valuation $v$ on $F$ such that $N_{S}=S O_{v}^{\times}$and $\left(v\left(F^{\times}\right): v(S)\right)=\left(F^{\times}: N_{S}\right)$.

Proof. Theorem 7.5 yields a valuation $v \in \operatorname{Val}\left(S, N_{S}\right)$. Thus $v$ is $S$-compatible and $O_{v}^{\times} \leq N_{S}$. By Proposition 7.1, $N_{S}=S O_{v}^{\times}$, whence the last equality.

In the exceptional case where $S$ is totally rigid, we have $N_{S}=\langle-1, S\rangle$ (Proposition 6.7(a)). Then the following slightly weaker version of Theorem 7.5 and Corollary 7.6 holds:

Proposition 7.7. Suppose that $\left(F^{\times}\right)^{p} \leq S \leq F^{\times}$is totally rigid. Then there exists an $S$-compatible valuation $v$ on $F$ such that $N_{S}=\langle-1, S\rangle$ is a subgroup of $S O_{v}^{\times}$of index dividing $p$. In particular, $\left(v\left(F^{\times}\right): v(S)\right) \geq\left(F^{\times}:\langle-1, S\rangle\right) / p$.

Proof. If there exists a valuation $v \in \operatorname{Val}\left(S, H_{S}\right)$, then $S \leq\langle-1, S\rangle \leq S O_{v}^{\times} \leq H_{S}$, so by Proposition $6.7(\mathrm{~b}),\left(S O_{v}^{\times}:\langle-1, S\rangle\right)\left|\left(H_{S}: S\right)\right| p$.

If $\operatorname{Val}\left(S, H_{S}\right)=\emptyset$, then by Proposition 3.3, $H_{S}=\langle-1, S\rangle, p=2, A_{S}=S$, and there exists a group $H_{S}<L \leq F^{\times}$such that $\left(L: H_{S}\right)=2$ and $\operatorname{Val}(S, L) \neq \emptyset$. Choose $v \in \operatorname{Val}(S, L)$. As $H_{S}=\langle-1, S\rangle \leq S O_{v}^{\times} \leq L$, we obtain $\left(S O_{v}^{\times}:\langle-1, S\rangle\right) \mid 2$ in this case as well.

For the last assertion of the theorem we compute:

$$
\begin{aligned}
\left(v\left(F^{\times}\right): v(S)\right)=\left(F^{\times}: S O_{v}^{\times}\right) & =\left(F^{\times}:\langle-1, S\rangle\right) /\left(S O_{v}^{\times}:\langle-1, S\rangle\right) \\
& \geq\left(F^{\times}:\langle-1, S\rangle\right) / p .
\end{aligned}
$$




\section{EXTENSIONS AND VALUATIONS}

We can now prove the following converse of Theorem 5.1.

Theorem 8.1. Let $p$ be a prime number, let $\left(F^{\times}\right)^{p} \leq S \leq F^{\times}$, and suppose that $p=2$ or $\left(F^{\times}: S\right)<\infty$. Furthermore, assume that $K_{*}^{M}(F) / S \cong \bar{A}[\Gamma]$ for some $\kappa$-structure $\bar{A} \neq \mathbf{0}, \kappa$ and an abelian group $\Gamma$ of exponent $p$. Then there exists an $S$-compatible valuation $v$ on $F$ such that $\left(v\left(F^{\times}\right): v(S)\right) \geq|\Gamma|$.

Proof. We have a sequence of group epimorphisms

$$
F^{\times} \rightarrow F^{\times} / S \stackrel{\sim}{\longrightarrow} \bar{A}_{1} \oplus \Gamma \rightarrow \Gamma
$$

the first and third maps being the obvious projections. It follows from Proposition 4.2 that $N_{S}$ is mapped trivially to $\Gamma$ under the composed epimorphism. Hence $\left(F^{\times}: N_{S}\right) \geq|\Gamma|$.

If $S$ is not totally rigid, then we are done by Corollary 7.6.

So suppose that $S$ is totally rigid. Then $K_{*}^{M}(F) / S \cong \bar{A}^{\prime}\left[\Gamma^{\prime}\right]$, where $\bar{A}^{\prime}$ is either $\mathbf{0}$ or $\kappa$ and $\Gamma^{\prime}$ is an abelian group of exponent $p$ (Proposition 6.1). The previous argument shows that $\left(F^{\times}: N_{S}\right) \geq\left|\Gamma^{\prime}\right|$. For an $S$-compatible valuation $v$ as in Proposition 7.7 we therefore have:

$$
\left(v\left(F^{\times}\right): v(S)\right) \geq\left(F^{\times}:\langle-1, S\rangle\right) / p=\left(F^{\times}: N_{S}\right) / p \geq\left|\Gamma^{\prime}\right| / p .
$$

Thus it suffices to show that $\left|\Gamma^{\prime}\right| / p \geq|\Gamma|$.

Indeed, we have $\bar{A}^{\prime}\left[\Gamma^{\prime}\right] \cong \bar{A}[\Gamma]$. If $\bar{A}^{\prime}=\mathbf{0}$, then $\Gamma^{\prime} \cong \bar{A}_{1} \oplus \Gamma$ and $\bar{A}_{1}$ is a non-trivial group of exponent $p$, so the claim is clear. On the other hand, if $\bar{A}^{\prime}=\kappa$, then $p=2$ and $\epsilon_{\bar{A}}^{r} \neq 0$ for all $r$. As $\bar{A} \neq \mathbf{0}, \kappa$ necessarily $\left|\bar{A}_{1}\right| \geq 4$. Hence $\kappa_{1} \oplus \Gamma^{\prime} \cong \bar{A}_{1} \oplus \Gamma$ gives $\left|\Gamma^{\prime}\right| / 2 \geq|\Gamma|$, as desired.

\section{FANS}

A fan on the field $F$ is a subset $T$ of $F$ such that $T \backslash\{0\}$ is a preordering on $F$, and for every $b \in F^{\times} \backslash(-T)$ one has $T+b T=T \cup b T$ ([BK], [L, Th. 5.5]). This notion, which has been extensively studied and used in the context of ordered fields, turns out to be essentially equivalent to the notion of a totally rigid preordering. Many other equivalent definitions can be given using the notions discussed so far.

Theorem 9.1. Let $S$ be a preordering on $F$. The following conditions are equivalent:

(a) $S$ is totally rigid;

(b) $S \cup\{0\}$ is a fan;

(c) for every $a \in F^{\times} \backslash(S \cup-S)$ one has $S-a S \subseteq S \cup-a S$;

(d) $A_{S}=S$;

(e) $H_{S}=\langle-1, S\rangle$;

(f) $N_{S}=\langle-1, S\rangle$;

(g) $K_{*}^{M}(F) / S \cong \kappa[\Gamma]$ for some abelian group $\Gamma$ of exponent 2 .

Proof. (a) $\Rightarrow(\mathrm{f})$ : $\quad$ This is Proposition 6.7(a).

(f) $\Rightarrow(\mathrm{d})$ : Here $p=2$, so Lemma 6.5(b) gives $A_{S} \subseteq N_{S}=\langle-1, S\rangle$. Furthermore, for $a \in-S$ we have $S-a S=S+S \subseteq S=S \cup-a S$, i.e., $a \notin A_{S}$. Thus $A_{S} \cap(-S)=\emptyset$.

$(\mathrm{d}) \Rightarrow(\mathrm{a})$ : This is Proposition 6.2 .

$(\mathrm{d}) \Leftrightarrow(\mathrm{e}),(\mathrm{d}) \Rightarrow(\mathrm{c})$ : $\quad$ Straightforward. 
(c) $\Rightarrow(\mathrm{b})$ : $\quad$ Set $T=S \cup\{0\}$ and take $b \in F^{\times} \backslash(-T)$ and $t_{1}, t_{2} \in T$. Let $c=t_{1}+b t_{2}$. We need to show that $c \in T \cup b T$. If $b \in S$, then $c \in T+T=T$, and we are done. Also, if $t_{1}=0$ or $t_{2}=0$, then the claim is clear. So suppose that $b \notin S$ and $t_{1}, t_{2} \in S$. Then $\pm b \in F^{\times} \backslash(S \cup-S)$. By (c), $c \in S+b S \subseteq S \cup b S \subseteq T \cup b T$.

(b) $\Rightarrow\left(\right.$ d): We always have $S \subseteq A_{S}$. Conversely, take $a \in F^{\times} \backslash S$ and set again $T=S \cup\{0\}$. (b) implies that $S-a S \subseteq T-a T=T \cup-a T$. But $0 \notin S-a S$, so $S-a S \in S \cup-a S$, i.e., $a \notin A_{S}$.

(a) $\Leftrightarrow(\mathrm{g})$ : This is Proposition 6.1(b).

\section{EXAMPLES OF TOTALly RIGID GROUPS}

The next list of examples shows that for $S$ totally rigid (so $N_{S}=\langle-1, S\rangle$ by Proposition 6.7(a)), the set $\operatorname{Val}(S,\langle-1, S\rangle)$ can be both empty or non-empty. In fact, each of these cases is possible when $-1 \in S$ as well as when $S \cup\{0\}$ is a fan. This shows that the assumption in Theorem 7.5(a) that $S$ is not totally rigid cannot be removed.

All our examples will be of the following general type: we take an appropriate field $\bar{F}$ of characteristic $\neq p$ and the ordered group $\Gamma=\mathbb{Z}^{m}$ with its lexicographic order, where $m$ is a cardinal number. We take $F=\bar{F}((\Gamma))$ to be the field of all formal power series $\alpha=\sum_{\gamma \in \Gamma} a_{\gamma} t^{\gamma}$ with $a_{\gamma} \in \bar{F}$ and well-ordered support $\left\{\gamma \in \Gamma \mid a_{\gamma} \neq 0\right\}$. As was shown by Hahn $[\mathrm{H}, F$ indeed forms a field with respect to the natural operations. We identify $\bar{F}$ with the subfield of all constant series in $F$. When $\alpha \neq 0$ one defines $v(\alpha)$ to be the minimal element of the support of $\alpha$. The map $v$ is a valuation on $F$ with value group $\Gamma$ and residue field $\bar{F}$. By a result of Krull [ $\mathrm{Kr}$, Satz 26], $(F, v)$ has no proper immediate extensions, whence it is Henselian. We will also take an appropriate subgroup $\left(\bar{F}^{\times}\right)^{p} \leq \bar{S} \leq \bar{F}^{\times}$and let $S=\left(F^{\times}\right)^{p} \bar{S}$. By Hensel's lemma, $1+\mathfrak{m}_{v} \leq\left(F^{\times}\right)^{p} \leq S$, i.e., $v$ is $\bar{S}$-compatible. Also $v(S)=p \Gamma$ and $\bar{S}_{v}=\bar{S}$, so by Theorem 5.1 :

$$
K_{*}^{M}(F) / S \cong\left(K_{*}^{M}(\bar{F}) / \bar{S}\right)\left[(\mathbb{Z} / p)^{m}\right] .
$$

Example 10.1. $S$ is totally rigid, $-1 \in S$, and $\operatorname{Val}(S, S) \neq \emptyset$. Take any $\bar{F}$ as above and $\bar{S}=\bar{F}^{\times}$. Then $K_{*}^{M}(\bar{F}) / \bar{S}=\mathbf{0}$, so $K_{*}^{M}(F) / S \cong \mathbf{0}\left[(\mathbb{Z} / p)^{m}\right]$. By Proposition 6.1(a), $S$ is totally rigid. The groups $O_{v}^{\times}$and $S \cap O_{v}^{\times}$have the same image $\bar{F}_{v}^{\times}$under the residue homomorphism. Hence $O_{v}^{\times} \leq\left(1+\mathfrak{m}_{v}\right)\left(S \cap O_{v}^{\times}\right) \leq S$, so $v \in \operatorname{Val}(S, S)$.

Example 10.2. $S \cup\{0\}$ is a fan and $\operatorname{Val}(S,\langle-1, S\rangle) \neq \emptyset$. Take $p=2, \bar{F}=\mathbb{R}$, and $\bar{S}=\left(\mathbb{R}^{\times}\right)^{2}$. Since $\bar{S}$ is an ordering on $\mathbb{R}$, we have $K_{*}^{M}(\bar{F}) / \bar{S}=\kappa$ (Proposition $5.2(\mathrm{a}))$. Thus $K_{*}^{M}(F) / S \cong \kappa\left[(\mathbb{Z} / 2)^{m}\right]$, so by Proposition $6.1(\mathrm{~b}), S$ is totally rigid. In light of Lemma 4.4, the Bockstein map here is injective. By [E5, Prop. 3.3] this implies that $S$ is a preordering, so by Theorem 9.1, $S \cup\{0\}$ is a fan.

Finally, the residue map induces an isomorphism $S O_{v}^{\times} / S \cong \bar{F}_{v}^{\times} / \bar{S}_{v}=\mathbb{R}^{\times} /\left(\mathbb{R}^{\times}\right)^{2}$. Hence $\left(S O_{v}^{\times}: S\right)=2=(\langle-1, S\rangle: S)$. As $\langle-1, S\rangle \leq S O_{v}^{\times}$, necessarily $\langle-1, S\rangle=$ $S O_{v}^{\times}$, i.e., $v \in \operatorname{Val}(S,\langle-1, S\rangle)$.

Example 10.3. $S$ totally rigid, $-1 \in S$, and $\operatorname{Val}(S, S)=\emptyset$. Dirichlet's theorem on primes in arithmetical progressions gives a prime number $l$ such that $l \equiv 1(\bmod$ $2 p)$. Take $\bar{F}=\mathbb{F}_{l}$ and $\bar{S}=\left(\mathbb{F}_{l}^{\times}\right)^{p}$. Then $S=\left(F^{\times}\right)^{p}$ and $-1 \in\left(\mathbb{F}_{l}^{\times}\right)^{p} \leq S$. The group $\mathbb{F}_{l}^{\times} /\left(\mathbb{F}_{l}^{\times}\right)^{p}$ is cyclic and non-trivial, whence of order $p$. Further, $K_{r}^{\bar{M}}\left(\mathbb{F}_{l}\right)=0$ for $r \geq 2$ [Mi, Example 1.5]. Therefore $K_{*}^{M}\left(\mathbb{F}_{l}\right) / \bar{S} \cong \mathbf{0}[\mathbb{Z} / p]$ (see Example 4.1(b)). 
It follows that

$$
K_{*}^{M}(F) / S \cong(\mathbf{0}[\mathbb{Z} / p])\left[(\mathbb{Z} / p)^{m}\right] \cong \mathbf{0}\left[(\mathbb{Z} / p)^{m+1}\right] .
$$

Conclude from Proposition 6.1(a) that $S$ is totally rigid.

Now suppose that $u \in \operatorname{Val}(S, S)$. To derive a contradiction we let $w$ be the finest common coarsening of $v$ and $u$. We claim that $O_{w}^{\times} \leq S$.

Indeed, if $u$ were strictly finer than $v$, then $O_{u / v}=O_{u} / \mathfrak{m}_{v}$ would be a proper valuation ring on $\bar{F}_{v}=\mathbb{F}_{l}$ (see $\S 2$ ), which is impossible. If $u$ is coarser than $v$, then $w=u$, so $O_{w}^{\times}=O_{u}^{\times} \leq S$. Finally, if $v$ and $u$ are incomparable, then Lemma 2.2 gives $O_{w}^{\times}=\left(1+\mathfrak{m}_{v}\right)\left(1+\mathfrak{m}_{u}\right) \leq S$. This proves the claim.

Consequently, $\bar{F}_{w}^{\times}=\bar{S}_{w}$. Using the comments at the end of $\S 2$ we now get the contradiction

$$
\mathbb{F}_{l}^{\times}=\bar{F}_{v}^{\times}=\left(\bar{F}_{w}^{\times}\right)_{v / w}=\left(\bar{S}_{w}\right)_{v / w}=\bar{S}_{v}=\left(\mathbb{F}_{l}^{\times}\right)^{p} .
$$

Example 10.4. $S \cup\{0\}$ is a fan and $\operatorname{Val}(S,\langle-1, S\rangle)=\emptyset$. Let $p=2$, let $\bar{F}=$ $\mathbb{Q}(\sqrt{2})$, and let $\bar{S}=\bar{S}_{1} \cap \bar{S}_{2}$, where $\bar{S}_{1}, \bar{S}_{2}$ are the distinct orderings on $\bar{F}$. By Proposition $5.2(\mathrm{~b}), K_{*}^{M}(\bar{F}) / \bar{S} \cong \kappa[\mathbb{Z} / 2]$. Therefore

$$
K_{*}^{M}(F) / S \cong(\kappa[\mathbb{Z} / 2])\left[(\mathbb{Z} / 2)^{m}\right] \cong \kappa\left[(\mathbb{Z} / 2)^{m+1}\right] .
$$

By Proposition 6.1(b), $S$ is totally rigid. Lemma 4.4 and [E5, Prop. 3.3] show again that $S$ is a preordering, i.e., $S \cup\{0\}$ is a fan.

Now suppose that $u \in \operatorname{Val}(S,\langle-1, S\rangle)$. To get a contradiction, we break the discussion as before into three cases.

If $v$ and $u$ are incomparable, then their finest common coarsening $w$ satisfies $-1 \in O_{w}^{\times}=\left(1+\mathfrak{m}_{v}\right)\left(1+\mathfrak{m}_{u}\right) \leq S$ (by Lemma 2.2$)$, which is not the case.

If $v$ is finer than $u$, then $O_{v}^{\times} \leq O_{u}^{\times} \leq\langle-1, S\rangle$. The residue map induces an isomorphism $S O_{v}^{\times} / S \cong \bar{F}_{v}^{\times} / \bar{S}_{v}=\bar{F}^{\times} / \bar{S}$. We get the obvious contradiction

$$
4=\left(\bar{F}^{\times}: \bar{S}\right)=\left(S O_{v}^{\times}: S\right) \mid(\langle-1, S\rangle: S)=2 .
$$

Finally suppose that $u$ is strictly finer than $v$. Then $u / v$ is a non-trivial valuation on $\bar{F}=\bar{F}_{v}$. As $1+\mathfrak{m}_{u} \leq S$ we have $1+\mathfrak{m}_{u / v} \leq \bar{S}_{v}=\bar{S}_{1} \cap \bar{S}_{2}$. Now any non-trivial valuation on the number field $\bar{F}$ is $\pi$-adic for some prime element $\pi$ (see $\mathrm{BO}$, Ch. VI, §1.4, Prop. 3]). Hence $(u / v)(l)>0$ for the prime number $l$ lying under $\pi$, so $1-l \in 1+\mathfrak{m}_{u / v} \leq \bar{S}_{1}$, and we again obtained a contradiction.

\section{REFERENCES}

[AEJ] J.K. Arason, R. Elman and B. Jacob, Rigid elements, valuations, and realization of Witt rings, J. Algebra 110 (1987), 449-467. MR0910395 (89a:11041)

[BaT] H. Bass and J. Tate, The Milnor ring of a global field, In: Algebraic $K$-Theory, Part II (H. Bass, ed.), (Battelle Institute Conference 1972; Springer Lect. Notes Math. 342, 1973, pp. 349-446. MR0442061 (56:449)

[BK] E. Becker and E. Köpping, Reduzierte quadratische Formen und Semiordnungen reeller Körper, Abh. Math. Sem. Univ. Hamburg 46 (1977), 143-177. MR0506028 (58:21934)

[Bo] N. Bourbaki, Commutative Algebra, Chapters 1-7, Springer, Berlin, 1989. MR0979760 (90a:13001)

[E1] I. Efrat, Construction of valuations from K-theory, Math. Res. Letters 6 (1999), 335-344. MR.1713134(2001i:12011)

[E2] I. Efrat, The local correspondence over absolute fields - an algebraic approach, Inter. Math. Res. Notices 2000:23 (2000), 1213-1223. MR.1809368 (2002g:12003)

[E3] I. Efrat, Demuškin fields with valuations, Math. Z. 243 (2003), 333-353. MR1961869 (2004d:11116) 
[E4] I. Efrat, A generalization of Marshall's equivalence relation, Trans. Amer. Math. Soc. 358 (2006), 2561-2577. MR 2204044

[E5] I. Efrat, Quotients of Milnor K-rings, orderings, and valuations, Pac. J. Math. 226 (2006), 259-276. MR2247864

[EF] I. Efrat and I. Fesenko, Fields Galois-equivalent to a local field of positive characteristic, Math. Res. Letters 6 (1999), 345-356. MR1713135(2001i:12004)

[En] O. Endler, Valuation Theory, Springer, Berlin, 1972. MR0357379 (50:9847)

[H] H. Hahn, Über die nichtarchimedischen Grössensysteme, Sitz.-Ber. d. Wiener Akad., Math.-Nat. Klasse, Abt. IIa 116 (1907), 601-653.

[HJ] Y.S. Hwang and B. Jacob, Brauer group analogues of results relating the Witt ring to valuations and Galois theory, Canad. J. Math. 47 (1995), 527-543. MR.1346152(97a:12004)

[J] B. Jacob, On the structure of pythagorean fields, J. Algebra 68 (1981), 247-267. MR0608534 (82g:12020)

[JWd] B. Jacob and A. Wadsworth, A new construction of noncrossed product algebras, Trans. Amer. Math. Soc. 293 (1986), 693-722. MR0816320 (87g:16027)

[Ka] B. Kahn, La conjecture de Milnor (d'aprés V. Voevodsky), Sém. Bourbaki 1996/97, Astérisque 245 (1997), 379-418. MR.1627119(2000a:19002)

[Ko] J. Koenigsmann, From p-rigid elements to valuations (with a Galois-characterisation of p-adic fields), J. reine angew. Math. 465 1995, 165-182. MR.1344135 (96m:12003)

[Kr] W. Krull, Allgemeine Bewertungstheorie, J. reine angew. Math. 167 (1931), 160-196.

[L] T.Y. Lam, Orderings, valuations and quadratic forms; Conf. Board of the Mathematical Sciences 52 , AMS, 1983. MR0714331 (85e:11024)

[Lg] S. Lang, Algebra, Addison-Wesley Publishing Company, Reading, Massachusetts, 1984. MR0783636 (86j:00003)

[MS] A.S. Merkurjev and A.A. Suslin, $K$-cohomology of Brauer-Severi varieties and the norm residue homomorphism, Math. USSR Izv. 21 (1983), 307-340; English translation (Russian).

[Mi] J. Milnor, Algebraic K-theory and quadratic forms, Invent. math. 9 (1970), 318-344. MR0260844(41:5465)

[NSW] J. Neukirch, A. Schmidt and K. Wingberg, Cohomology of Number Fields, Springer, Berlin-Heidelberg, 2000. MR.1737196 (2000j:11168)

[R] P. Ribenboim, Théorie des valuations, Les Presses de l'Université de Montréal, Montréal, 1968. MR0249425(40:2670)

[S] K. Szymiczek, Quadratic forms over fields, Dissertationes Math. (Rosprawy Mat.) 152 (1977). MR0450199 (56:8495)

[Wd] A.R. Wadsworth, p-henselian fields: K-theory, Galois cohomology, and graded Witt rings, Pac. J. Math. 105 (1983), 473-496. MR0691616 (84m:12026)

[Wr1] R. Ware, Valuation rings and rigid elements in fields, Canad. J. Math. 33 (1981), 13381355. MR0645230 (83i:10028)

[Wr2] R. Ware, Galois groups of maximal p-extensions, Trans. Amer. Math. Soc. 333 (1992), 721-728. MR.1061780 (92m:12008)

Department of Mathematics, Ben-Gurion University of the Negev, P.O. Box 653, BE'ER-SHEVA 84105, ISRAEL

E-mail address: efrat@math.bgu.ac.il 